\title{
Screening for Coxiella burnetii infection during pregnancy: pros and cons according to the Wilson and Jungner criteria
}

J M Munster (J.Munster@umcg.nl)1,2,3, L M Steggerda ${ }^{1}$, A C Leenders ${ }^{4}$, J G Aarnoudse ${ }^{2}$, E Hak Ha $^{1,3}$

1. University of Groningen, University Centre for Pharmacy, PharmacoEpidemiology and PharmacoEconomics, Groningen, the Netherlands

2. University of Groningen, University Medical Centre Groningen, Department of Obstetrics and Gynaecology, Groningen, the Netherlands

3. University of Groningen, University Medical Centre Groningen, Department of Epidemiology, Groningen, the Netherlands

4. Jeroen Bosch Hospital, Department of Medical Microbiology and Infection Prevention, 's-Hertogenbosch, the Netherlands

Citation style for this article:

Munster JM, Steggerda LM, Leenders AC, Aarnoudse JG, Hak E. Screening for Coxiella burnetii infection during pregnancy: pros and cons according to the Wilson and Jungner criteria.

Euro Surveill. 2012;17(3):pii=20061. Available online: http://www.eurosurveillance.org/ViewArticle.aspx?Articleld=20061

Article published on 19 January 2012

In Europe the incidence of human $Q$ fever has dramatically increased over the previous years. Untreated infections with Coxiella burnetii, the causal agent of $Q$ fever, have been associated with both obstetric and maternal complications. The majority of pregnant women with a $C$. burnetii infection remain asymptomatic, hence screening could be of value to prevent unwanted outcomes in this high-risk group. We applied the updated Wilson and Jungner criteria to review the evidence for routine screening for $C$. burnetii infection during pregnancy. Since much uncertainty remains about the incidence, clinical consequences, diagnostics and treatment of $C$. burnetii infection during pregnancy, routine screening for $C$. burnetii infection during pregnancy should not be recommended. Rigorous studies to assess the effectiveness of $C$. burnetii screening are warranted.

\section{Introduction}

Infections during pregnancy may cause a threat to both maternal and foetal health, even if the infected pregnant woman herself remains asymptomatic [1]. Therefore, routine screening at 12 weeks of gestation is being offered to all Dutch pregnant women for human immunodeficiency virus (HIV), Treponema pallidum and hepatitis B virus (HBV). The incidence of human $Q$ fever, a zoonosis caused by Coxiella burnetii, showed an enormous increase in the Netherlands and other European countries over the past few years [2]. Since there is evidence for infection-associated obstetric and maternal complications, $C$. burnetii infection poses a potential risk to pregnant women and their (unborn) children [3]. Most of the pregnant women with a C. burnetii infection remain asymptomatic [4]. Therefore routine screening has been put forward for early detection and treatment in this group, but scientific evidence about the usefulness of such an intensive program is lacking. In this review we applied the Wilson and Jungner criteria according to the World Health Organization to scrutinise the available evidence for routine screening for $C$. burnetii infection during pregnancy. These criteria were developed over 40 years ago but are still of great value in decision making around screening policies [5]. The criteria centre on the problem caused by the infection or disease, the screening population, the test and the treatment, and the costs. As newer policy tools, especially concerning genetic screening, have been suggested [6], we also integrated the emerging criteria which are applicable to our research question. A review of the literature was done by searching PubMed and the references of included papers. Our search was limited to studies in English or Dutch. The search strategy included the keywords ' $Q$ fever' or 'Coxiella burnetii' and keywords related to the criteria ('incidence' or 'prevalence' or 'pregnancy' or 'risk factors' or 'diagnosis' or 'treatment' or 'costs'). Our overall aim was to examine the evidence base for routine C. burnetii screening among pregnant women in highrisk areas for $\mathrm{Q}$ fever all over Europe.

\section{The problem}

Terminology used in the scientific literature concerning ' $Q$ fever' is diverse and therefore direct comparisons of epidemiological studies should be performed with caution. ' $Q$ fever' is commonly referred to the symptomatic disease, including symptoms such as fever, hepatitis or pneumonia in combination with positive antibody titres or polymerase chain reaction (PCR). The terms 'C. burnetii infection' and 'presence of antibodies' are more often used in the context of asymptomatic disease, for example, in prevalence studies.

\section{Is Coxiella burnetii infection during} pregnancy an important health problem?

Prior to 2007 Q fever was uncommon in Europe [2], except from some local outbreaks such as the outbreak 
in Germany in spring 2005, causing 331 cases [7]. In the Netherlands around 10 to 30 cases have been notified each year since 1977. Between 2007 and 2009 the numbers briskly increased to over 2,300 cases in 2009, the highest number ever reported in the literature [8]. Veterinary outbreaks on several dairy goat and sheep farms in the southern parts of the Netherlands are held responsible for this increase. In 2009 and 2010 it was decided to implement extensive measures such as vaccinating and culling of thousands of animals [8]. As a result, the number of human $Q$ fever cases decreased rapidly to around 500 cases by the end of 2010, which is still considerable and may indicate an endemic stage [9]. Also other European countries, such as Belgium, Cyprus and Germany have reported an increasing number of cases since 2007 , albeit to a smaller extent [2].

The prevalence of $Q$ fever among pregnant women is unknown. Recently published data from the Netherlands showed a prevalence of immunoglobulin (Ig)M, suggesting recent infection with $C$. burnetii, in $3.4 \%$ of 1,646 tested serum samples from pregnant women in Q fever high-risk areas [10]. In a cohort study from Canada, $3.8 \%$ of parturient women had evidence of previous exposure to $C$. burnetii (presence of IgG phase I and/or II). These women had, in contrast to the Dutch seropositive women [10], a higher risk for adverse pregnancy outcomes, in terms of premature delivery and prior or current neonatal death, compared with seronegative women [11]. A milestone hospitalbased study from France showed that $81 \%$ of the pregnant women with untreated $\mathrm{Q}$ fever had a miscarriage, premature delivery, intrauterine growth restriction or foetal death. Furthermore, chronic $Q$ fever occurred in $50 \%$ of the cases, of whom $10 \%$ developed C. burnetii endocarditis [3]. These figures are alarming, but need to be cautiously interpreted as the retrospective design covering many years may have led to some overestimation of risks. Certainly, this study together with the prevalence studies emphasise that $C$. burnetii infection is a potential threat to pregnant women.

\section{Is there a latent or early symptomatic stage?}

Up to $90 \%$ of infected pregnant women remain asymptomatic [4]. Therefore, early detection, before obstetric complications and maternal chronic $Q$ fever have occurred, enables treatment that may prevent complications due to $C$. burnetii infection [3].

\section{Is the natural history of Coxiella burnetii infection adequately understood?}

C. burnetii is a small gram-negative intracellular living bacterium. The main route of transmission is the respiratory route, in which alveolar macrophages in the lungs are the first cells to be infected [12]. Furthermore, the placenta seems to be a target organ since placentitis has been described in both animals and humans $[3,13]$. After the primary infection, $C$. burnetii has the ability to induce chronic infections. It is hypothesised that, besides the liver, bone, heart valves and mural endocardium [14], the uterus could be a site of latent infection, hence reactivation during pregnancy can occur $[3,11]$.

The pathogenesis of obstetric complications following infection is not completely understood; immune complexes may cause vasculitis and vascular thrombosis, which in turn may lead to the placental insufficiency and subsequent obstetric complications [15]. Also, direct transplacental transmission by $C$. burnetii may cause foetal death [16]. Obstetric complications occur significantly more often in patients who are infected during the first trimester of pregnancy than in those infected later [3].

Not only have acute infections been associated with obstetric complications, but also previous infections seem to increase the risk [11]. There is no good explanation for this association besides the hypothesis of intrauterine latency of the infection [11]. In all, the natural history of $C$. burnetii infection among pregnant women is not completely understood.

\section{The screening population}

Since the $Q$ fever incidence largely varies between regions (see for example the situation in the Netherlands, figure), the population for routine screening should be limited to pregnant women living in highrisk $\mathrm{Q}$ fever areas. Women living within a five-kilometre zone around a dairy goat or dairy sheep farm affected by $C$. burnetii-related abortion waves have the highest risk of contracting an infection, however, still $41 \%$ of the Dutch cases in 2009 lived outside of these areas [8]. Whether these cases visited the five-kilometre zones is unclear. Therefore, if introduced, routine screening of all pregnant women would be advisable in areas with a high incidence (e.g. >50/100,000 inhabitants). So, with a good surveillance system, the screening population can be accurately defined. Screening of specific groups at risk, e.g. pregnant women with occupational hazard for $\mathrm{Q}$ fever or with complicated pregnancies can also be considered, but is beyond the scope of this study discussing routine screening of a total population.

Similar to other screening programs during pregnancy, eligible women have to be counselled about the benefits and possible consequences of the screening (i.e. long-term antibiotic treatment and hospital birth instead of home birth in case of an acute infection, stress induced by awareness of infectious diseases during pregnancy) to be able to make an informed choice about participation.

Is there an agreed policy on whom to treat as patients? All phases of $C$. burnetii infection during pregnancy have been associated with adverse pregnancy outcome. However, evidence for the benefits of antibiotic treatment is only available in patients with acute and chronic Q fever [3]. Whether antibiotic treatment prevents complications in women with asymptomatic seropositivity needs to be investigated. 
Is case finding a continuing process and not a 'once and for all' project?

If introduced, screening for $C$. burnetii infection should be performed during each pregnancy since the infection can be contracted at any moment and reactivation during pregnancy of a previous infection may occur $[3,11]$. Therefore case finding is a continuing process.

\section{The test and the treatment}

\section{Is there a suitable test?}

There are several accurate methods to diagnose C. burnetii infection, including culture, PCR and serology, of which serology is most suitable for screening [17]. However, the performance of these tests during pregnancy is unknown. In the general population, indirect immunofluorescence assay (IFA) is the reference method $[17,18]$. Since one of the characteristics of $C$. burnetii is antigenetic phase variation, antibodies against two phases of antigens can be detected. All types of antibodies have their own timeframe of appearance, therefore distinguishing previous, acute and chronic infections is possible $[12,18]$. As already mentioned, test characteristics during pregnancy are unknown. From other infectious diseases we know that false-positive serological results occur quite often

\section{FIGURE}

Human Q fever incidence per 100,000 inhabitants per municipality in the Netherlands, 1 January-12 August 2009

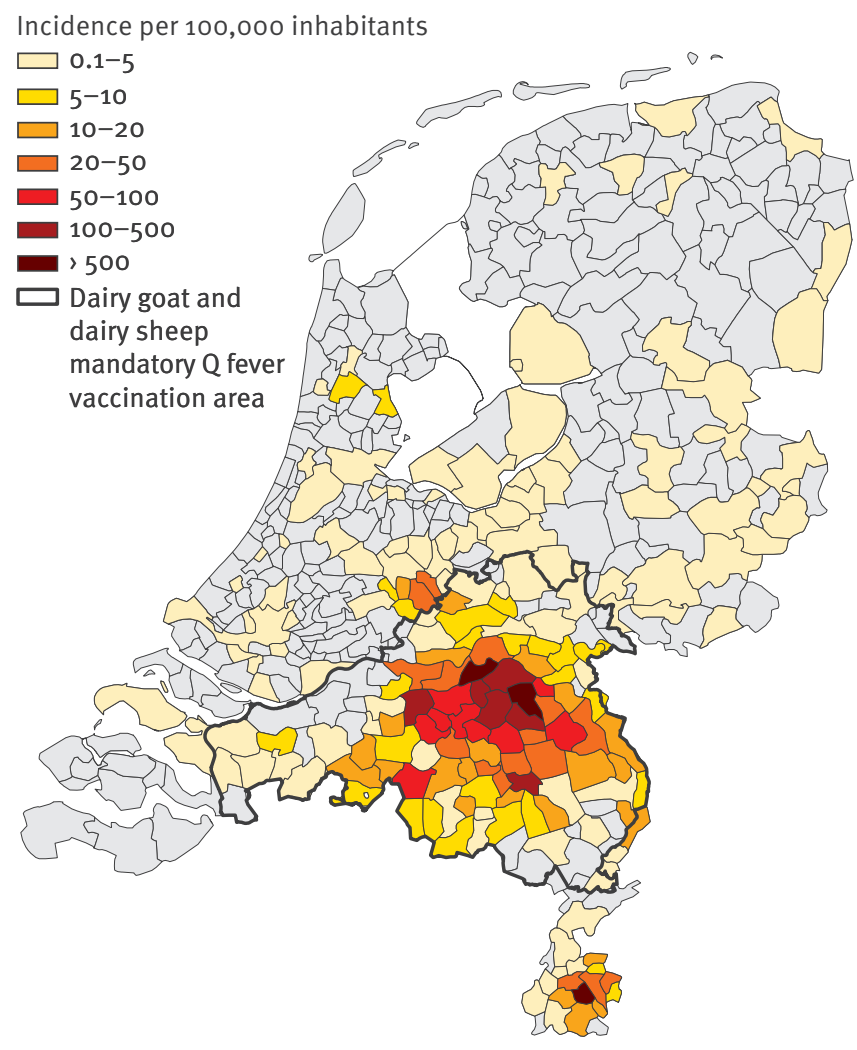

Incidences are based on symptomatic (fever, pneumonia and/or hepatitis), laboratory-confirmed Q fever cases.

Source: National Institute for Public Health and the Environment (RIVM). during pregnancy [19]. Furthermore, with respect to sensitivity and specificity, there is an ongoing debate about which cut-off values to use, especially because there are many different commercial and in-house methods. In all, more research needs to be performed with respect to serological screening for $C$. burnetii during pregnancy before routine screening can be implemented.

Is the test acceptable to the population?

Acceptance of the test can be expected since only one blood sample is necessary, which can be obtained by venepuncture combined with the screening for other infectious diseases around 12 weeks of pregnancy. An advantage of testing in the first trimester is the possibility of early counselling and treatment during the most vulnerable phase of pregnancy [3]. However, with early screening, infections later in pregnancy would be missed. Timing of the screening needs to be further investigated, also taking into account a seasonal variation in C. burnetii spreading [9].

\section{Is there an accepted treatment for} patients with recognised disease?

First choice treatment for $\mathrm{Q}$ fever among the general population is a 14-day antibiotic treatment with doxycycline or fluoroquinolone [12]. However, both agents are contraindicated during pregnancy. Long-term treatment with cotrimoxazole has been suggested to be the treatment of choice during pregnancy [3]. However, use of cotrimoxazole during pregnancy has not been fully investigated yet. Pharmacological activity of this drug could cause folic acid depletion in the foetus [20]. Furthermore, neonatal hyperbilirubinemia has been described when used prior to delivery. However, these risks turned out to be small in large groups of pregnant women with HIV who received prophylactic cotrimoxazole therapy during pregnancy [21]. In all, more evidence for the best treatment option during pregnancy is needed.

\section{Are there facilities for diagnosis \\ and treatment available?}

Since screening for other infectious diseases during pregnancy is already routinely performed, adding C. burnetii screening will be relatively straightforward. In the Netherlands, as in other Western countries, several laboratories have facilities to perform $C$. burnetii serology. Quality assessments should be performed on a regular basis. Treatment and follow-up of positively screened women should be performed by obstetricians, infectious disease specialists and medical microbiologist, who should receive additional training on diagnostics and treatment of $C$. burnetii infection during pregnancy.

\section{The costs}

Are the costs of case finding economically balanced in relation to possible expenditure on medical care as a whole? 
Outcomes of cost-effectiveness models are not available yet and input data are required. Screening with IFA and antibiotic treatment are relatively cheap, though referral for treatment and hospital birth may induce high costs since around $25 \%$ of the deliveries in the Netherlands normally take place at home [22].

The adapted Wilson and Jungner criteria, addressed in this study are summarised in the table.

\section{Conclusion}

According to the adapted Wilson and Jungner criteria (Table), the currently available evidence is insufficient to promote routine screening for $C$. burnetii infection during pregnancy in high-risk $Q$ fever areas. Because of potential bias in the studies reported so far, there is too much uncertainty about the consequences of untreated $C$. burnetii infection during pregnancy. There is also no consensus about the screening method and treatment. Furthermore, $\mathrm{Q}$ fever incidence rates highly affect the effectiveness of screening. Therefore the candidate populations for screening are not static and should be identified based on epidemiological criteria. Finally, besides screening, there are other methods to prevent $C$. burnetii related complications, for example human vaccination [23]. Overall, more evidence about the effectiveness of a $C$. burnetii screening program, in addition to other $\mathrm{Q}$ fever prevention and control measures taken by the European countries, is needed before this infection will become a candidate for routine screening during pregnancy.

\section{Disclosure statement}

EH and JGA are members of the Health Council of the Netherlands on a non-profit base.

\section{Acknowledgments}

The authors thank Ben Bom from the National Institute for Public Health and the Environment (RIVM) for compiling the figure in this manuscript.

\section{References}

1. Gilbert GL. 1: Infections in pregnant women. Med J Aust. 2002;176(5):229-36.

2. European Centre for Disease Prevention and Control (ECDC). Annual epidemiological report on communicable diseases in Europe 2010. Stockholm: ECDC; 2010. Available from: http:// www.ecdc.europa.eu/en/publications/Publications/1011 SUR Annual Epidemiological Report on_Communicable Diseases_in_Europe.pdf

\section{TABLE}

Wilson and Jungner criteria and emerging criteria for disease screening

\section{Wilson and Jungner criteria and emerging criteria for disease screening}

\section{The problem}

\begin{tabular}{|c|c|}
\hline The condition sought should be an important health problem. & Not certain \\
\hline There should be a latent or early symptomatic stage. & Yes \\
\hline The natural history of the condition should be adequately understood. & Not certain \\
\hline The screening program should respond to a recognised need. & Not certain \\
\hline The objectives of screening should be defined at the outset. & Yes \\
\hline \multicolumn{2}{|l|}{ The screening population } \\
\hline There should be an agreed policy on whom to treat as patients. & Not certain \\
\hline Case finding should be a continuing process and not a 'once and for all' project. & Yes \\
\hline There should be a defined target population. & Yes \\
\hline The program should ensure informed choice, confidentiality and respect for autonomy. & Yes \\
\hline The program should promote equity and access to screening for the entire target population. & Not applicable \\
\hline \multicolumn{2}{|l|}{ The test and the treatment } \\
\hline There should be an accepted treatment for patients with recognised disease. & Not certain \\
\hline Facilities for diagnosis and treatment should be available. & Yes \\
\hline There should be a suitable test or examination. & Not certain \\
\hline The test should be acceptable to the population. & Yes \\
\hline There should be quality assurance, with mechanisms to minimise potential risks of screening. & Not certain \\
\hline \multicolumn{2}{|l|}{ The costs } \\
\hline The costs of case finding should be economically balanced in relation to possible expenditure on medical care as a whole. & Not certain \\
\hline \multicolumn{2}{|l|}{ Overall } \\
\hline There should be scientific evidence of screening program effectiveness. & No \\
\hline The program should integrate education, testing, clinical services and program management. & Not applicable \\
\hline Program evaluation should be planned from the outset. & Not applicable \\
\hline The overall benefits of screening should outweigh the harm. & Not certain \\
\hline
\end{tabular}

Wilson and Jungner criteria for disease screening adopted by the World Health Organization [5], combined with the emerging screening criteria proposed over the past 40 years [6] (italic). 
3. Carcopino X, Raoult D, Bretelle F, Boubli L, Stein A. Managing $Q$ fever during pregnancy: the benefits of long-term cotrimoxazole therapy. Clin Infect Dis. 2007;45(5):548-55.

4. Tissot-Dupont H, Vaillant V, Rey S, Raoult D. Role of sex, age, previous valve lesion and pregnancy in the clinical expression and outcome of $Q$ fever after a large outbreak. Clin Infect Dis. 2007;44(2):232-7.

5. Wilson JMG, Jungner G. Principles and practice of screening for disease. World Health Organization public health papers. 1968;34. Geneva: World Health Organization; 1968. Available from: http://whqlibdoc.who.int/php/WHO_PHP_34.pdf

6. Andermann A, Blancquaert I, Beauchamp S, Déry V. Revisiting Wilson and Jungner in the genomic age: a review of screening criteria over the past 40 years. Bull World Health Organ. 2008;86(4):317-9.

7. Gilsdorf A, Kroh C, Grimm S, Jensen E, Wagner-Wiening C, Alpers K. Large Q fever outbreak due to sheep farming near residential areas, Germany, 2005. Epidemiol Infect. 2008;136(8):1084-7.

8. Roest HI, Tilburg JJ, Van der Hoek W, Vellema P, van

Zijderveld FG, Klaassen $\mathrm{CH}$, et al. The $\mathrm{Q}$ fever epidemic in The Netherlands: history, onset, response and reflection. Epidemiol Infect. 2011;139(1):1-12.

9. National Institute for Public Health and the Environment (RIVM). Q-koorts [Q fever]. Bilthoven: RIVM. [Accessed 27 Jun 2011]. Dutch. Available from: http://www.rivm.nl/ Onderwerpen/Ziekten_Aandoeningen/Q/Q_koorts

10. Van der Hoek W, Meekelenkamp JCE, Leenders ACAP, Wijers N, Notermans DW, Hukkelhoven CWPM. Antibodies against Coxiella burnetii and pregnancy outcome during the 2007$2008 \mathrm{Q}$ fever outbreaks in The Netherlands. BMC Infect Dis. 2011;11:44.

11. Langley JM, Marrie TJ, Le Blanc JC, Almudevar A, Resch L, Raoult D. Coxiella burnetii seropositivity in parturient women is associated with adverse pregnancy outcomes. Am J Obstet Gynecol. 2003;189(1):228-32.

12. Maurin M, Raoult D. Q fever. Clin Microbiol Rev. 1999;12(4):518-53.

13. Woude W, Dercksen DP. Abortion and stillbirth among dairy goats as a consequence of Coxiella burnetii. Tijdschr Diergeneeskd. 2007;132(23):908-11. Dutch.

14. Harris RJ, Storm PA, Lloyd A, Arens M, Marmion BP. Long-term persistence of Coxiella burnetii in the host after primary $Q$ fever. Epidemiol Infect. 2000;124(3):543-9.

15. Sánchez J, Souriauy A, Buendía AJ, Arricau-Bouvery N, Martínez CM, Salinas J, et al. Experimental Coxiella burnetii infection in pregnant goats: a histopathological and immunohistochemical study. J Comp Pathol. 2006;135(2-3):108-15.

16. Stein A, Raoult D. Q fever during pregnancy: a public health problem in Southern France. Clin Infect Dis. 1998;27(3):592-6.

17. Fournier P, Marrie TJ, Raoult D. Diagnosis of Q fever. J Clin Microbiol. 1998:36(7):1823-34.

18. Dupont HT, Thirion X, Raoult D. Q fever serology: cutoff determination for microimmunofluorescence. Clin Diagn Lab Immunol. 1994;1(2):189-96.

19. Jones JL, Krueger A, Schulkin J, Schantz PM. Toxoplasmosis prevention and testing in pregnancy, survey of obstetriciangynaecologists. Zoonoses Public Health. 2010;57(1):27-33.

20. Hernández-Díaz S, Werler MM, Walker AM, Mitchell AA. Folic acid antagonists during pregnancy and the risk of birth defects. N Engl J Med. 2000;343(22):1608-14.

21. Forna F, McConnell M, Kitabire FN, Homsy J, Brooks JT, Mermin J, et al. Systematic review of the safety of trimethoprimsulfamethoxazole for prophylaxis in HIV-infected pregnant women: implications for resource-limited settings. AIDS Rev. 2006;8(1);24-36.

22. Anthony S, Amelink-Verburg MP, Jacobusse GW, van der Pal-de Bruin KM. De Thuisbevalling in Nederland 19952002. Rapportage over de jaren 2001-2002 [Report on home delivery in the Netherlands 2001-2002]. Bilthoven: The Netherlands Perinatal Registry; June 2005. Dutch. Available from: http://www.perinatreg.nl/uploads/153/99/Rapportage Thuisbevalling_2001-2002.pdf

23. Gidding HF, Wallace C, Lawrence GL, McIntyre PB. Australia's national Q fever vaccination program. Vaccine. 2009;27(14):2037-41. 